\title{
Quantum Statistical Properties of Resonant Radiation Scattered on Excited Systems
}

\author{
Boris A. Veklenko \\ Joint Institute for High Temperature of Russian Academy of Science, Moscow, Russia \\ E-mail:VeklenkoBA@yandex.ru \\ Received April 15, 2010; revised May 21, 2010; accepted June 10, 2010
}

\begin{abstract}
The scattering of resonant radiation on an excited atom is considered. It is shown that the scattering cross section calculated with the help of quantum theory of radiation is five times larger than the one calculated using semi-classical theory. The quantum theory predicts, in general, the change in internal quantum statistical properties of light due to the scattering processes on excited atoms.
\end{abstract}

Keywords: Quantum Theory, Semi-Classical Theory, Resonant Radiation

\section{Introduction}

The quantum excited systems possess remarkable properties. They manifest themselves most prominently in lasers and masers, which were created in the middle of the last century. The theory of these devices was elaborated by W. Lamb [1] on the base of a semi classical theory of radiation which deals with classical electromagnetic field. Later the quantum theory was proposed [2]. It is possible to state omitting the fluctuations properties that both the semi classical and the quantum theories result practically in the same results for quantum means values. Such a fact resulted in overestimation of the applicability of the semi-classical theory. In 1966 year, Ch. Koester predicted the effect of light enhancement [3] by selective reflection of resonant radiation from excited media. All efforts of quantitative explaining this effect on the base of semi-classical theory of radiation discussed in monograph [4] were unsuccessful $[5,6]$. It was shown later that quantum field theory should be used instead [7], but the mathematically problems on this way occurred very difficult [8]. The consequences of such a theory manifest themselves on a macroscopic level. The correct description of stimulated radiation plays an especial role when the resonant reflection of light from excited media is considered. Nevertheless, there are recent works [9] which make use the semiclassical theory and Fresnel's formulae to describe the reflection of light from enhanced media.

Much attention has been paid recently to the effect of the enhanced transmission of light through the metallic films $[10,11]$. There is no agreement between theory and experiment. It is believed that the enhancement of radiation may be explained through the interaction of light with induced standing surface-plasmon waves. Thus we deal with effects of stimulate radiation, which means that one should use the quantum field theory.

Examples shown above made us revise the theory of resonant radiation scattering on excited systems. The conventional perturbation technique is not adequate to describe the resonant scattering and it is necessary to sum up (Dyson summation) the infinitely long subsets of Feynman's ladder diagrams. It was V. Weisskopff and E. Wigner who constructed such a theory for the first time by considering the interaction of resonant radiation with atomic systems [12]. Such a summation of Feynman's diagram proved to be useful for the shape of spectrum line of resonant radiation and effects of resonant light scattering on non excited systems. The difficulties emerge in the theory of combined resonant scattering processes when one of the photons after stimulation emission of excited atom undergoes of elastic scattering on the ground state of the same atom. Such combined scattering is non-analytic in charge. The summation of the Feynman's diagrams like this one is not performed up to now [8]. We propose indirect way to estimate this sum.

Present work demonstrates insufficiency WeisskopffWigner's method and Dyson's method of summation Feynman's ladder diagrams for the calculations the cross-sections of light scattering on resonant excited systems and failure of semi-classical theory of radiation.

Let the resonant radiation scatters on some system the initial state of which in interaction representation is described by wave function $\psi_{0}$. The total wave function of electromagnetic field and scattering system is denoted as $\Psi$. The expansion of such function over a base of scattering system wave function $\psi_{i}$ is 


$$
\Psi=f_{0} \psi_{0}+\sum_{i \neq 0} f_{i} \psi_{i}=f_{0} \psi_{0}+\left(\Psi-f_{0} \psi_{0}\right) .
$$

The term containing $\psi_{0}$ is written separately. The scalar product due to orthogonality of scatter's wave functions

$$
\left\langle f_{0} \psi_{0} \mid \Psi-f_{0} \psi_{0}\right\rangle=0
$$

is equal to zero. Assume that the incident light is in quantum coherent state [13] and its quantum mean electrical strength is not equal to zero $\left\langle\hat{\mathcal{E}}^{v}(\mathbf{r}, t)\right\rangle \neq 0$ in all space points $\mathbf{r}$ at arbitrary instant of time $t$. We are interesting in quantum mean value of operator $\hat{\varepsilon}^{v}$ of the reflected light

$$
\begin{aligned}
& \left\langle\hat{\mathcal{E}}^{v}(\mathbf{r}, t)\right\rangle=\left\langle\Psi\left|\hat{\mathcal{E}}^{v}(\mathbf{r}, t)\right| \Psi\right\rangle= \\
& \left\langle f_{0} \psi_{0}\left|\hat{\mathcal{E}}^{v}(\mathbf{r}, t)\right| f_{0} \psi_{0}\right\rangle+\left\langle\Psi-f_{0} \psi_{0}\left|\hat{\mathcal{E}}^{v}(\mathbf{r}, t)\right| \Psi-f_{0} \psi_{0}\right\rangle(1) \\
& =\mathcal{E}^{v(c)}+\boldsymbol{\varepsilon}^{v(n)}
\end{aligned}
$$

We state that the first term of the right hand side of Equation (1) describes the so-called coherent scattering channel with medium returning to the initial quantum state after scattering (e.g. elastic scattering). The second term of the right hand side of Equation (1) describes the non-coherent scattering processes with the medium changing initial quantum state (Compton scattering, Raman scattering and induced radiation of light). The latter is very important. We stress once again that the coherent Heisenber-Kramers scattering and induced radiation of light are described by different scattering channels. It means that if the scattering media consisted only of the non-excited atoms the first term of Equation (1) would describe the coherent Heisenberg-Kramers scattering while the second one would describe the diffusion scattering. If the excited atoms are present in the medium then due to the induced radiation processes it is impossible to avoid the presence of the non-coherent channel even if only the selective scattering is under our investigation. The total measured electrical strength $\left\langle\hat{\mathcal{E}}^{v}(\mathbf{r}, t)\right\rangle$, that is the left hand part of Equation (1), may be evaluated separately using the semi-classical theory of radiation if one neglects the fluctuation optical processes and their influence on $\left\langle\hat{\varepsilon}^{v}(\mathbf{r}, t)\right\rangle$. The region of validity of the semi-classical theory of radiation is very large but it does not mean that $\left\langle\hat{\varepsilon}^{v}(\mathbf{r}, t)\right\rangle$ describes the bilinear field characteristics.

Let us consider the energy characteristics of electromagnetic field described by normal operator product $\left\langle\hat{N}\left(\hat{\varepsilon}^{v}\right)^{2}\right\rangle$. Such value should be estimated from below using the following procedure. One takes into account that

$$
\hat{\varepsilon}^{v}(\mathbf{r}, t)=i \sum_{\mathbf{k} \lambda} \sqrt{\frac{\hbar c k}{2 V}} e_{\mathbf{k} \lambda}^{v}\left(\hat{\alpha}_{\mathbf{k} \lambda} e^{i \mathbf{k r}-i c k t}-\hat{\alpha}_{\mathbf{k} \lambda}^{+} e^{-i \mathbf{k r}+i c k t}\right),
$$

where $\hat{\alpha}_{\mathbf{k} \lambda}$ and $\hat{\alpha}_{\mathbf{k} \lambda}^{+}$are the annihilation and creation photon operators in states describing by wave vector $\mathbf{k}$ and polarization index $\lambda$. These operators obey the conventional commutation relations

$$
\left[\hat{\alpha}_{\mathbf{k} \lambda} ; \hat{\alpha}_{\mathbf{k}^{\prime} \lambda^{\prime}}^{+}\right]=\delta_{\mathbf{k k}^{\prime}} \delta_{\lambda \lambda^{\prime}}
$$

Consider electromagnetic field as a transverse one $(\lambda=1,2), \mathbf{e}_{\mathbf{k} \lambda}$ denotes the unite linear polarization vectors, $V$ is the quantization volume. Since the operators $\hat{\alpha}_{\mathbf{k} \lambda}$ and $\hat{\alpha}_{\mathbf{k} \lambda}^{+}$are mutual conjugate than

$\left\langle\sum_{\mathbf{k}^{\prime} \lambda^{\prime}} e_{\mathbf{k}^{\prime} \lambda^{\prime}}^{v} \sqrt{k^{\prime}}\left(\hat{\alpha}_{\mathbf{k}^{\prime} \lambda^{\prime}}^{+}-\left\langle\hat{\alpha}_{\mathbf{k}^{\prime} \lambda^{\prime}}^{+}\right\rangle\right) \sum_{\mathbf{k} \lambda} e_{\mathbf{k} \lambda}^{v} \sqrt{k}\left(\hat{\alpha}_{\mathbf{k} \lambda}-\left\langle\hat{\alpha}_{\mathbf{k} \lambda}\right\rangle\right) e^{i\left(\mathbf{k}-\mathbf{k}^{\prime}\right) \mathbf{r}-i c\left(k-k^{\prime}\right) t}\right\rangle \geq 0$

Now

$$
\begin{aligned}
& \sum_{\mathbf{k} \mathbf{k}^{\prime} \lambda \lambda^{\prime}} \sqrt{k k^{\prime}} e_{\mathbf{k} \lambda}^{v} e_{\mathbf{k}^{\prime} \lambda^{\prime}}^{v}\left\langle\hat{\alpha}_{\mathbf{k}^{\prime} \lambda^{\prime}}^{+} \hat{\alpha}_{\mathbf{k} \lambda}\right\rangle e^{i\left(\mathbf{k}-\mathbf{k}^{\prime}\right) \mathbf{r}-i c\left(k-k^{\prime}\right) t} \geq \\
& \sum_{\mathbf{k} \mathbf{k}^{\prime} \lambda \lambda^{\prime}} \sqrt{k k^{\prime}} e_{\mathbf{k} \lambda}^{v} e_{\mathbf{k}^{\prime} \lambda^{\prime}}^{v}\left\langle\hat{\alpha}_{\mathbf{k} \lambda}\right\rangle\left\langle\hat{\alpha}_{\mathbf{k}^{\prime} \lambda^{\prime}}^{+}\right\rangle e^{i\left(\mathbf{k}-\mathbf{k}^{\prime}\right) \mathbf{r}-i c\left(k-k^{\prime}\right) t}
\end{aligned}
$$

If the electromagnetic field possesses the characteristic frequency $\omega_{0}$ and characteristic wave length $\lambda_{0}$ and we are interesting in time and space values much larger then $1 / \omega_{0}$ and $\lambda_{0}$ the following inequality occurs

$$
\begin{aligned}
& \sum_{\mathbf{k} \mathbf{k}^{\prime} \lambda \lambda^{\prime}} \sqrt{k k^{\prime}} e_{\mathbf{k} \lambda}^{v} e_{\mathbf{k}^{\prime} \lambda^{\prime}}^{v}\left\langle\hat{\alpha}_{\mathbf{k}^{\prime} \lambda^{\prime}}^{+} \hat{\alpha}_{\mathbf{k} \lambda}\right\rangle e^{i\left(\mathbf{k}-\mathbf{k}^{\prime}\right) \mathbf{r}-i c\left(k-k^{\prime}\right) t}+ \\
& \sum_{\mathbf{k} \mathbf{k}^{\prime} \lambda \lambda^{\prime}} \sqrt{k k^{\prime}} e_{\mathbf{k} \lambda}^{v} e_{\mathbf{k}^{\prime} \lambda^{\prime}}^{v}\left\langle\hat{\alpha}_{\mathbf{k}^{\prime} \lambda}^{+} \hat{\alpha}_{\mathbf{k}^{\prime} \lambda^{\prime}}\right\rangle e^{-i\left(\mathbf{k}-\mathbf{k}^{\prime}\right) \mathbf{r}+i c\left(k-k^{\prime}\right) t}>> \\
& \sum_{\mathbf{k} \mathbf{k}^{\prime} \lambda \lambda^{\prime}} \sqrt{k k^{\prime}} e_{\mathbf{k} \lambda}^{v} e_{\mathbf{k}^{\prime} \lambda^{\prime}}^{v}\left\langle\hat{\alpha}_{\mathbf{k} \lambda} \hat{\alpha}_{\mathbf{k}^{\prime} \lambda^{\prime}}\right\rangle e^{i\left(\mathbf{k}+\mathbf{k}^{\prime}\right) \mathbf{r}-i c\left(k+k^{\prime}\right) t}+ \\
& \sum_{\mathbf{k} \mathbf{k}^{\prime} \lambda \lambda^{\prime}} \sqrt{k k^{\prime}} e_{\mathbf{k} \lambda}^{v} e_{\mathbf{k}^{\prime} \lambda^{\prime}}^{v}\left\langle\hat{\alpha}_{\mathbf{k} \lambda}^{+} \hat{\alpha}_{\mathbf{k}^{\prime} \lambda^{\prime}}^{+}\right\rangle e^{-i\left(\mathbf{k}+\mathbf{k}^{\prime}\right) \mathbf{r}+i c\left(k+k^{\prime}\right) t}
\end{aligned}
$$

Now it is non difficult to see that

$$
\begin{gathered}
\left\langle\hat{N}\left(\hat{\mathcal{E}}^{v}(\mathbf{r}, t)\right)^{2}\right\rangle \approx \\
\sum_{\mathbf{k} \mathbf{k}^{\prime} \lambda \lambda^{\prime}} \frac{\hbar c}{V} \sqrt{k k^{\prime}} e_{\mathbf{k} \lambda}^{v} e_{\mathbf{k}^{\prime} \lambda^{\prime}}^{v}\left\langle\hat{\alpha}_{\mathbf{k} \lambda}^{+} \hat{\alpha}_{\mathbf{k}^{\prime} \lambda^{\prime}}\right\rangle e^{-i\left(\mathbf{k}-\mathbf{k}^{\prime}\right) \mathbf{r}+i c k\left(t-t^{\prime}\right)} \geq \\
\sum_{\mathbf{k} \lambda} \frac{\hbar c}{V} \sqrt{k k^{\prime}} e_{\mathbf{k} \lambda^{v}}^{v} e_{\mathbf{k}^{\prime} \lambda^{\prime}}^{v}\left\langle\hat{\alpha}_{\mathbf{k} \lambda}^{+}\right\rangle\left\langle\hat{\alpha}_{\mathbf{k}^{\prime} \lambda^{\prime}}\right\rangle e^{-i\left(\mathbf{k}-\mathbf{k}^{\prime}\right) \mathbf{r}+i c k\left(t-t^{\prime}\right)} \\
\approx\left\langle\hat{\mathcal{E}}^{v}(\mathbf{r}, t)\right\rangle^{2}
\end{gathered}
$$

Thus $\left\langle\hat{\varepsilon}^{v}\right\rangle$ proposes the opportunity to estimate 
$\left\langle\hat{N}\left(\hat{\varepsilon}^{v}\right)^{2}\right\rangle$ from below. The validity of obtained inequality does not depend on particular quantum state on which the averaging is performed and does nothing to do with perturbation theory. But if such inequality is applied to each term of right hand site of

$$
\begin{aligned}
& \left\langle\hat{N}\left(\hat{\mathcal{E}}^{v}\right)^{2}\right\rangle=\left\langle f_{0} \psi_{0}\left|\hat{N}\left(\hat{\mathcal{E}}^{v}\right)^{2}\right| f_{0} \psi_{0}\right\rangle+ \\
& \left\langle\Psi-f_{0} \psi_{0}\left|\hat{N}\left(\hat{\mathcal{E}}^{v}\right)^{2}\right| \Psi-f_{0} \psi_{0}\right\rangle
\end{aligned}
$$

We find that

$$
\begin{aligned}
& \left\langle\hat{N}\left(\hat{\mathcal{E}}^{v}\right)^{2}\right\rangle \geq \\
& \left\langle f_{0} \psi_{0}\left|\hat{\mathcal{E}}^{v}\right| f_{0} \psi_{0}\right\rangle^{2}+\left\langle\Psi-f_{0} \psi_{0}\left|\hat{\mathcal{E}}^{v}\right| \Psi-f_{0} \psi_{0}\right\rangle^{2}
\end{aligned}
$$

The last formula can be rewritten in as

$$
\begin{aligned}
& \left\langle\hat{N}\left(\hat{\boldsymbol{\varepsilon}}^{v}\right)^{2}\right\rangle \geq \\
& \left\langle f_{0} \psi_{0}\left|\hat{\boldsymbol{\varepsilon}}^{v}\right| f_{0} \psi_{0}\right\rangle^{2}+\left(\left\langle\Psi\left|\hat{\boldsymbol{\varepsilon}}^{v}\right| \Psi\right\rangle-\left\langle f_{0} \psi_{0}\left|\hat{\boldsymbol{\varepsilon}}^{v}\right| f_{0} \psi_{0}\right\rangle\right)^{2}
\end{aligned}
$$

That stresses the importance of the coherent scattering channel when the scattered light is not classical and.

$$
\left\langle\hat{N}\left(\hat{\mathcal{E}}^{v}\right)^{2}\right\rangle \neq\left\langle\hat{\mathcal{E}}^{v}\right\rangle^{2} \text {. }
$$

Inequality (4) allows to estimate $\left\langle\hat{N}\left(\hat{\mathcal{E}}^{v}\right)^{2}\right\rangle$ in the semi-classical approximation. The value $\left\langle\Psi\left|\hat{\mathcal{E}}^{v}\right| \Psi\right\rangle=\mathcal{E}^{v}$ can be calculated using the conventional semi-classical theory operating with non quantum electromagnetic field. The calculation $\left\langle f_{0} \psi_{0}\left|\hat{\mathcal{E}}^{v}\right| f_{0} \psi_{0}\right\rangle=\mathcal{E}^{v(c)}$ can be performed using only the coherent scattering channel. Even in extensive media such procedure may be performed with the help of wave functions [14]. Thus one can avoid of matrix density formalism specific for non coherent scattering channel.

\section{Principal Equations}

Let the electromagnetic field scatters on an atom situated at a point with radius-vector $\mathbf{R}$ and for the sake of simplicity possesses only one orbital electron with coordinate $\mathbf{r}$. Let the atom possesses only two energy levels. Zeeman`s sublevels with different magnetic numbers are possible. Let the frequency of incident radiation $\omega$ is in a quasi resonance $\left|\omega_{0}-\omega\right|<<\omega_{0}+\omega$ with the atom transition frequency $\omega_{0}$. Let Schroedinger equation for atom and radiation is as follows

$$
i \hbar \frac{\partial \Psi}{\partial t}=\left(\hat{H}_{a}+\hat{H}_{p h}+\hat{H}^{\prime}\right) \Psi,
$$

where

$$
\begin{gathered}
\hat{H}_{a}=\int \hat{\psi}^{+}(\mathbf{r})\left(\frac{\hat{p}_{\mathbf{r}}^{2}}{2 m}+U(\mathbf{r}-\mathbf{R})\right) \hat{\psi}(\mathbf{r}) d \mathbf{r} \\
\hat{H}^{\prime}=-\frac{e}{m c} \int \hat{\psi}^{+}(\mathbf{r}) \hat{p}_{\mathbf{r}}^{v} \hat{A}^{v}(\mathbf{r}) \hat{\psi}(\mathbf{r}) d \mathbf{r}
\end{gathered}
$$

are the Hamiltonian of the non-interacting atoms and an interaction Hamiltonian in Schroedinger representation. Than

$$
\begin{gathered}
\hat{\psi}(\mathbf{r})=\sum_{j} \psi_{j}(\mathbf{r}-\mathbf{R}) \hat{b}_{j}, \quad \hat{\psi}^{+}(\mathbf{r})=\sum_{j} \psi_{j}^{*}(\mathbf{r}-\mathbf{R}) \hat{b}_{j}^{+}, \\
\hat{p}_{\mathbf{r}}^{v}=-i \hbar \nabla_{\mathbf{r}}^{v} .
\end{gathered}
$$

The following communitation relations are assumed

$$
\left[\hat{b}_{j} ; \hat{b}_{j^{\prime}}^{+}\right]=\delta_{j j^{\prime}}
$$

for the electron creation operator $\hat{b}_{j}^{+}$and annihilation operator $\hat{b}_{j}$ in the state described by wave function $\psi_{j}$. The particular form of communication relations in our case of one electron in the atom does not play any role. By $U(\mathbf{r}-\mathbf{R})$ we denote the potential energy of atom electron. The Einstein summation rule is assumed over all repeating indices $v$ throughout the paper. The Hamiltonian of free electromagnetic field and vector-potential operator are as follows

$$
\begin{gathered}
\hat{H}_{p h}=\sum_{\mathbf{k} \lambda} \hbar c k \hat{\alpha}_{\mathbf{k} \lambda}^{+} \hat{\alpha}_{\mathbf{k} \lambda} \\
\hat{A}^{v}(\mathbf{r})=\sum_{\mathbf{k} \lambda} \sqrt{\frac{\hbar c}{2 k V}} e_{\mathbf{k} \lambda}^{v}\left(\hat{\alpha}_{\mathbf{k} \lambda} e^{i \mathbf{k r}}+\hat{\alpha}_{\mathbf{k} \lambda}^{+} e^{-i \mathbf{k r}}\right)
\end{gathered}
$$

In order to realize the calculation project mentioned in introduction we switch to the interaction representation with the help of unitary operator

$$
\hat{U}(t)=\exp \left(\frac{1}{i \hbar}\left(\hat{H}_{a}+\hat{H}_{p h}\right) t\right) \text {. }
$$

In this picture

$$
\begin{gathered}
\Psi(t)=\hat{S}\left(t, t_{0}\right) \Psi_{0}, \hat{S}\left(t, t_{0}\right)=\hat{T} \exp \left(\frac{1}{i \hbar} \int_{-\infty}^{t} \hat{H}^{\prime}\left(t^{\prime}\right) d t^{\prime}\right) \\
\hat{H}^{\prime}(t)=-\frac{e}{m c} \int \hat{\psi}^{+}(x) \hat{p}_{\mathbf{r}}^{v} \hat{A}^{v}(x) \hat{\psi}(x) d \mathbf{r}, \quad x=\{\mathbf{r}, t\}, \\
\hat{\psi}(x)=\sum_{j} \psi_{j}(\mathbf{r}-\mathbf{R}) e^{-i \frac{\varepsilon_{j}}{\hbar} t} \hat{b}_{j} \\
\hat{\psi}^{+}(x)=\sum_{j} \psi_{j}^{*}(\mathbf{r}-\mathbf{R}) e^{i \frac{\varepsilon_{j}}{\hbar} t} \hat{b}_{j}^{+} .
\end{gathered}
$$

where $\hat{S}\left(t, t_{0}\right)$ is the scattering operator, $\varepsilon_{j}$ is the atomic energy in state $\psi_{j}, \hat{T}$ is the time-ordering operator and 


$$
\begin{aligned}
& \hat{A}^{v}(x)=\hat{A}^{v(+)}(x)+\hat{A}^{v(-)}(x)= \\
& \sum_{\mathbf{k} \lambda} \sqrt{\frac{\hbar c}{2 k V}} e_{\mathbf{k} \lambda}^{v} \hat{\alpha}_{\mathbf{k} \lambda} e^{i \mathbf{k}-i k c t}+\sum_{\mathbf{k} \lambda} \sqrt{\frac{\hbar c}{2 k V}} e_{\mathbf{k} \lambda}^{v} \hat{\alpha}_{\mathbf{k} \lambda}^{+} e^{-i \mathbf{k r}+i k c t}
\end{aligned}
$$

\section{Coherent Scattering Channel}

Suppose that the initial state of the field was described by $\left(\mathbf{k}_{0}, \lambda_{0}\right)$ and was in quantum coherent state [13]

$$
f_{0}^{0}=e^{-\frac{1}{2}|\alpha|^{2}} \sum_{n} \frac{\left(\alpha \hat{\alpha}_{\mathbf{k}_{0} \lambda_{0}}\right)^{n}}{\sqrt{n !}}|0\rangle .
$$

The amplitude of initial radiation was

$$
\begin{aligned}
& \left\langle\Psi_{0}\left|\hat{A}^{v}(x)\right| \Psi_{0}\right\rangle=\left\langle\begin{array}{l}
0 \\
f_{0}
\end{array} \psi_{0}\left|\hat{A}^{v}(x)\right| \begin{array}{l}
0 \\
f_{0} \psi_{0}
\end{array}\right\rangle= \\
& e_{\mathbf{k}_{0} \lambda_{0}}^{v}\left(a_{\mathbf{k}_{0} \lambda_{0}} e^{i \mathbf{k}_{0} \mathbf{r}-i k_{0} c t}+a_{\mathbf{k}_{0} \lambda_{0}}^{*} e^{-i \mathbf{k}_{0} \mathbf{r}+i k_{0} c t}\right), \\
& a_{\mathbf{k}_{0} \lambda_{0}}=\sqrt{\frac{\hbar c}{2 k V}} \alpha .
\end{aligned}
$$

We are interested in the radiation amplitude after scattering in second order of perturbation technique. The problem of Feynman's diagrams summation will be discussed below. In Equation (5) it is sufficient to consider the sum

$$
\hat{S}=1+\hat{S}^{(1)}+\hat{S}^{(2)}+\hat{S}^{(3)},
$$

where

$$
\begin{gathered}
\hat{S}^{(1)}=\frac{1}{i \hbar} \int \hat{H}^{\prime}\left(t^{\prime}\right) d t^{\prime} \\
\hat{S}^{(2)}=\frac{\hat{T}}{2(i \hbar)^{2}}\left(\int \hat{H}^{\prime}\left(t^{\prime}\right) d t^{\prime}\right)^{2}, \\
\hat{S}^{(3)}=\frac{\hat{T}}{3 !(i \hbar)^{3}}\left(\int \hat{H}^{\prime}\left(t^{\prime}\right) d t^{\prime}\right)^{3} .
\end{gathered}
$$

If the photon scatters in the coherent channel then the atom rests in initial state. So in second order of perturbation technique we are interested in the construction

$$
\mathcal{A}^{v(c)}(x)=\left\langle\Psi_{0}\left|\hat{A}^{v}(x) \hat{S}^{(2)}\right| \Psi_{0}\right\rangle+\text { c.c. }
$$

We change the time-ordering product of the atom operators by the normal ordering one. For the scattering operator $\hat{S}^{(2)}$ we get

$\hat{S}^{(2)}=$

$\left(\frac{e}{m c}\right)^{2} \frac{\hat{T}_{A}}{i \hbar} \int \hat{\psi}^{+}\left(x_{1}\right) \hat{p}_{\mathbf{r}_{1}}^{v_{1}} \hat{A}^{v_{1}}\left(x_{1}\right) G_{r}\left(x_{1}, x_{2}\right) \hat{p}_{\mathbf{r}_{2}}^{v_{2}} \hat{A}^{v_{2}}\left(x_{2}\right) \hat{\psi}\left(x_{2}\right) d x_{1} d x_{2}$ where $\hat{T}_{A}$ is the time-ordering operator acting only on the electromagnetic field operators and

$$
(\hat{T}-\hat{N}) \hat{\psi}(x) \hat{\psi}^{+}\left(x^{\prime}\right)=i \hbar G_{r}\left(x_{1}, x_{2}\right),
$$

$$
\begin{aligned}
& G_{r}\left(x_{1}, x_{2}\right)= \\
& \sum_{j} \int \psi_{j}\left(\mathbf{r}_{1}-\mathbf{R}\right) \psi_{j}^{*}\left(\mathbf{r}_{2}-\mathbf{R}\right) e^{-\frac{E}{\hbar}\left(t_{1}-t_{2}\right)} G_{r}^{j}\left(\frac{E}{\hbar}\right) \frac{d E}{2 \pi \hbar} \\
& G_{r}^{j}\left(\frac{E}{\hbar}\right)=\frac{1}{E-\varepsilon_{j}+i 0}
\end{aligned}
$$

If the atom undergoes the action of external random fields the finite width of its energy levels can be taken into account by replacing the term $+i 0$ by $+i \gamma_{j} / 2$ with the same sign because it is governed by the causality principle. The same result follows from summing up (Dyson summation) the ladder Feynman`s diagrams for excited atoms due to their interaction with electromagnetic vacuum. For the same reason formula (8) can be written as

$$
G_{r}^{j}\left(\frac{E}{\hbar}\right)=\frac{1}{E-\varepsilon_{j}+i \frac{\gamma_{j}}{2}} .
$$

without specifying the value $\gamma_{j}$. We take into account that,

$$
\begin{aligned}
& \hat{T}_{A} \hat{A}^{v_{1}}\left(x_{1}\right) \hat{A}^{v_{2}}\left(x_{2}\right)= \\
& i \hbar D^{v_{1} v_{2}}\left(x_{1}, x_{2}\right)+\hat{N} \hat{A}^{v_{1}}\left(x_{1}\right) \hat{A}^{v_{2}}\left(x_{2}\right)
\end{aligned}
$$

where $D^{v_{1} v_{2}}\left(x_{1}, x_{2}\right)$ is not the operator function. The first term in (9) does not play any role in electromagnetic field scattering process. Finitely

$$
\begin{aligned}
& \hat{S}^{(2)}=\frac{1}{i \hbar}\left(\frac{e}{m c}\right)^{2} \int \hat{\psi}^{+}\left(x_{1}\right) \hat{p}_{\mathbf{r}_{1}}^{v_{1}} G_{r}\left(x_{1}, x_{2}\right) \hat{p}_{\mathbf{r}_{2}}^{v_{2}} \\
& {\left[\hat{A}^{v_{2}(-)}\left(x_{2}\right) \hat{A}^{v_{1}(+)}\left(x_{1}\right)+\hat{A}^{v_{1}(-)}\left(x_{1}\right) \hat{A}^{v_{2}(+)}\left(x_{2}\right)\right] \hat{\psi}\left(x_{2}\right) d x_{1} d x_{2}}
\end{aligned}
$$

The right hand side terms of this equality are responsible on scattering processes of electromagnetic field by both the non excited atom and excited one.

\subsection{Scattering on Non-Excited Atom}

Substituting (6) and (8) into Equation (10) and taken the limit $t \rightarrow \infty$, we find

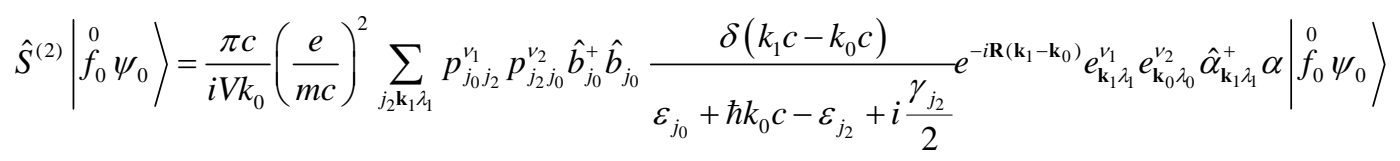


Through $j_{0}$ one denote here the quantum number of initial state of atom. In dipole approximation

$$
p_{j_{0} j_{2}}^{v}=\int \psi_{j_{0}}^{*}(\boldsymbol{\rho}) \hat{p}_{\boldsymbol{\rho}}^{v} \psi(\boldsymbol{\rho}) d \boldsymbol{\rho} .
$$

The limit $t \rightarrow \infty$ is not necessary but it makes the calculations simpler. According to (7) we need to calculate the construction

$$
\mathscr{A}^{v(c)}(x)=\left\langle\begin{array}{l}
0 \\
f_{0}
\end{array} \psi_{0}\left|\hat{A}^{v(+)}(x) \hat{S}^{(2)}\right| \begin{array}{l}
0 \\
f_{0}
\end{array} \psi_{0}\right\rangle+\text { c.c. }
$$

Let us use the following equalities connecting any smooth function $f(k)$ and limits $V \rightarrow \infty, \mathbf{r}-\mathbf{R} \mid \rightarrow \infty$

$$
\begin{aligned}
& \sum_{\mathbf{k}_{1} \lambda_{1}} e_{\mathbf{k}_{1} \lambda_{1}}^{v} e_{\mathbf{k}_{1} \lambda_{1}}^{v_{1}} \delta\left(k_{1} c-k_{0} c\right) e^{i \mathbf{k}_{1}(\mathbf{r}-\mathbf{R})} f\left(k_{1}\right)= \\
& A^{v(c)}(x)=-\frac{1}{4 \pi}\left(\frac{e}{m c}\right)^{2} \frac{\delta\left(\delta_{v v_{1}}-n^{v} n^{v_{1}}\right)}{|\mathbf{r}-\mathbf{R}|} a_{\mathbf{k}_{0} \lambda_{0}} e^{i \mathbf{k}_{0} \mathbf{R}} \sum_{j_{2}} p_{j_{0} j_{2}}^{v_{1}} p_{j_{2} j_{0}}^{v_{2}} \frac{1}{\hbar c k_{0}-\omega_{0} \hbar+i \frac{\gamma_{j_{2}}}{2}} e_{\mathbf{k}_{0} \lambda_{0}}^{v_{2}} e^{i k_{0}|\mathbf{r}-\mathbf{R}|-i k_{0} c t}+c . c .
\end{aligned}
$$

\subsection{Scattering on Excited Atom}

$$
\mathcal{A}^{v(c)}(x)=-\frac{1}{4 \pi}\left(\frac{e}{m c}\right)^{2} \frac{\left(\delta_{v v_{2}}-n^{v} n^{\nu_{2}}\right)}{|\mathbf{r}-\mathbf{R}|} a_{\mathbf{k}_{0} \lambda_{0}} e^{i \mathbf{k}_{0} \mathbf{R}} \sum_{j_{2}} p_{j_{0} j_{2}}^{v_{1}} p_{j_{2} j_{0}}^{v_{2}} \frac{1}{\omega_{0} \hbar-\hbar c k_{0}+i \frac{\gamma_{j_{2}}}{2}} e_{\mathbf{k}_{0} \lambda_{0}}^{v_{1}} e^{i k_{0}|\mathbf{r}-\mathbf{R}|-i k_{0} c t}+c . c .
$$

If one takes into account the width of atom's energy level in state described by $\psi_{j_{0}}$ than it is necessary to replace $\gamma_{j_{2}} \rightarrow \gamma=\gamma_{j_{0}}+\gamma_{j_{2}}$ in Equation (12). The validity of Equations (11) and (12) are restricted by domain $\gamma_{r} / \gamma<<1$ where $\gamma_{r}$ is the radiation width of excited state of atom.

\section{Non Coherent Scattering Channel}

The second order perturbation technique gets

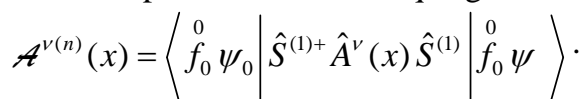

Or by taken into account only the scattering processes we have in explicit form

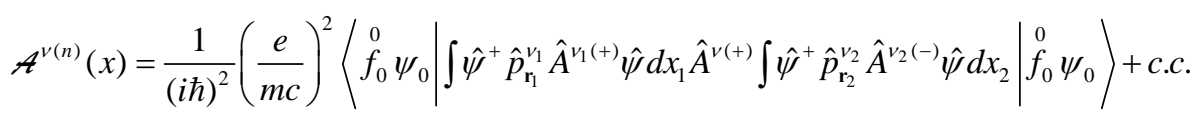

Following the procedures described in part 3.1 we have

$$
\mathscr{A}^{v(n)}(x)=\frac{i}{2 \hbar}\left(\frac{e}{m c}\right)^{2} \frac{\left(\delta_{v v_{2}}-n^{v} n^{\nu_{2}}\right)}{|\mathbf{r}-\mathbf{R}|} a_{\mathbf{k}_{0} \lambda_{0}} e^{i \mathbf{k}_{0} \mathbf{R}} \sum_{j_{2}} p_{j_{0} j_{2}}^{v_{1}} p_{j_{2} j_{0}}^{v_{2}} \delta\left(\frac{\varepsilon_{j_{0}}-\varepsilon_{j_{2}}}{\hbar}-k_{0} C\right) e_{\mathbf{k}_{0} \lambda_{0}}^{v_{1}} e^{i k_{0}|\mathbf{r}-\mathbf{R}|-i k_{0} c t}+\text { c.C. }
$$

If we take into account the finite width of atom energy

level than in formula (13) it is necessary to change

$$
\delta\left(\frac{\varepsilon_{j_{0}}-\varepsilon_{j_{2}}}{\hbar}-k_{0} c\right) \rightarrow-\frac{\hbar}{2 \pi i}\left(\frac{1}{\varepsilon_{j_{0}}-\varepsilon_{j_{2}}-k_{0} c+i \frac{\gamma}{2}}-\frac{1}{\varepsilon_{j_{0}}-\varepsilon_{j_{2}}-k_{0} c-i \frac{\gamma}{2}}\right)
$$


Let us find now the total amplitude of electromagnetic field scattered by excited atom

$$
\mathcal{A}^{v}(x)=\mathcal{A}^{v(c)}(x)+\mathcal{A}^{v(n)}(x)=-\frac{1}{4 \pi}\left(\frac{e}{m c}\right)^{2} \frac{\left(\delta_{v v_{2}}-n^{v} n^{\nu_{2}}\right)}{|\mathbf{r}-\mathbf{R}|} a_{\mathbf{k}_{0} \lambda_{0}} e^{i \mathbf{k}_{0} \mathbf{R}} \sum_{j_{2}} p_{j_{0} j_{2}}^{\nu_{1}} p_{j_{2} j_{0}}^{\nu_{2}} \frac{1}{\omega_{0} \hbar-\hbar c k_{0}-i \frac{\gamma}{2}} e_{\mathbf{k}_{0} \lambda_{0}}^{v_{1}} e^{i k_{0}|\mathbf{r}-\mathbf{R}|-i k_{0} c t}+\text { c.c. }
$$

\section{Semi-Classical Theory of Radiation}

The set equations for field operators $\stackrel{v}{\psi}(x)$ and $A^{v}(x)$ in Heisenberg representation is the following

$$
\begin{aligned}
& i \hbar \frac{\partial \psi(x)}{\partial t}= \\
& \left(\frac{\hat{p}_{\mathbf{r}}^{2}}{2 m}+U(\mathbf{r}-\mathbf{R})-\frac{e}{m c} \stackrel{\vee}{A^{v}}(x) \hat{p}_{\mathbf{r}}^{v}\right) \stackrel{v}{\psi}(x) \\
& \stackrel{v}{v}(x)=-\frac{1}{c} j^{v}(x), \quad \stackrel{j^{v}}{v}(x)=\frac{e}{2 m}\left(\stackrel{v}{\psi^{+}} \hat{p}_{\mathrm{r}}^{v} \stackrel{v}{\psi}+\hat{p}_{\mathrm{r}}^{v *} \psi^{+} \cdot v\right) .
\end{aligned}
$$

This set equations is equivalent to the one mentioned at part 3. Now

$$
\begin{aligned}
& \stackrel{\vee}{A^{v}}(x)=\stackrel{\vee}{A^{v 0}}(x)-\frac{e}{m c} \int \Delta_{r}^{v v_{1}}\left(x, x_{1}\right) \stackrel{v}{\psi} \psi^{+}\left(x_{1}\right) \hat{p}_{\mathbf{r}_{1}}^{v_{1}} \stackrel{v}{\psi}\left(x_{1}\right) d x_{1} \cdot(15) \\
& \stackrel{v}{A^{v}}(x)=\stackrel{v}{A^{v 0}}(x)+\left(\frac{e}{m c}\right)^{2} \int \Delta_{r}^{v v_{1}}\left(x, x_{1}\right) \stackrel{v}{\hat{\psi}^{+}}\left(x_{1}\right) \hat{p}_{\mathbf{r}_{1}}^{v_{1}} G_{r}\left(x_{1}, x_{2}\right) \hat{p}_{\mathbf{r}_{2}}^{v_{2}} \hat{A}^{v_{2}}\left(x_{2}\right) \stackrel{v}{\hat{\psi}}\left(x_{2}\right) d x_{1} d x_{2}+\text { H.c. }
\end{aligned}
$$

For the mean values, the same result can be obtained either by averaging (18) with subsequent breaking up the
Here $A^{v 0}(x)$ is given by the formula (6) and

$$
\begin{aligned}
& \Delta_{r}^{v v_{1}}\left(x, x_{1}\right)=\frac{1}{i \hbar}\left[A^{v 0}(x) ; A^{v v_{1} 0}\left(x_{1}\right)\right] \vartheta\left(t-t_{1}\right)= \\
& -\frac{c}{4 \pi} \frac{\delta_{v v_{1}}-n^{v} n^{v_{1}}}{\left|\mathbf{r}-\mathbf{r}_{1}\right|} \delta\left(\left|\mathbf{r}-\mathbf{r}_{1}\right|-c\left(t-t_{1}\right)\right) \quad,\left|\mathbf{r}-\mathbf{r}_{1}\right| \rightarrow \infty .
\end{aligned}
$$

We are interested in the second order perturbation expansion. This mean that the $\psi(x)$ operator has be evaluated in the first order of perturbation technique

$$
\stackrel{\vee}{\psi}(x)=\stackrel{\vee}{\psi^{0}(x)-\frac{e}{m c} \int G_{r}\left(x, x_{1}\right)} \hat{p}_{\mathbf{r}_{1}}^{v_{1}} \stackrel{\vee}{A^{v_{1}}}\left(x_{1}\right) \stackrel{v}{\psi}\left(x_{1}\right) d x_{1} .
$$

$$
\left\langle A^{v}\left(x_{2}\right)\right\rangle \rightarrow \stackrel{0}{v_{2}} A^{\nu_{2}}\left(x_{2}\right)=\left\langle\Psi_{0}\left|A^{\nu_{2}}\left(x_{2}\right)\right| \Psi_{0}\right\rangle=e_{\mathbf{k}_{0} \lambda_{0}}^{v_{2}}\left(a_{\mathbf{k}_{0} \lambda_{0}} e^{i \mathbf{k}_{0} \mathbf{r}_{2}-i k_{0} c t_{2}}+a_{\mathbf{k}_{0} \lambda_{0}}^{*} e^{-i \mathbf{k}_{0} \mathbf{r}_{2}+i k_{0} c_{2}}\right)
$$

for the scattered field we have

$$
\mathscr{A}^{v}(x)=-\frac{1}{4 \pi}\left(\frac{e}{m c}\right)^{2} \frac{\left(\delta_{v v_{1}}-n^{v} n^{v_{1}}\right)}{|\mathbf{r}-\mathbf{R}|} a_{\mathbf{k}_{0} \lambda_{0}} e^{ \pm i \mathbf{k}_{0} \mathbf{R}} \sum_{j_{2}} p_{j_{0} j_{2}}^{v_{1}} p_{j_{2} j_{0}}^{v_{2}} \frac{1}{\varepsilon_{j_{0}}-\varepsilon_{j_{2}} \pm \hbar c k_{0}+i 0} e_{\mathbf{k}_{0} \lambda_{0}}^{v_{2}} e^{ \pm i k_{0}|\mathbf{r}-\mathbf{R}| \mp i k_{0} c t}+c . c .
$$

The upper sign describes the scattering of electromagnetic field on the non-excited atom while the low one describes the scattering on excited atom. One should take into account the width of atomic energy level by replacing in dominator $+i 0$ with $+i \gamma / 2=+i\left(\gamma_{j_{0}}+\gamma_{j_{2}}\right) / 2$.

By comparing Equation (19) with Equations (11) and (14) we find that in the approximation we used both the quantum theory and the semi-classical theory result in the same expressions for the scattered amplitude $A^{v}(x)$. Namely the necessary coinciding in such results leads to the equality of constants $\gamma$ in Formulas (19), (11) and (14).

\section{Bilinear Field Charasteristics}

In this part we are interesting in the following construction shown in introduction

$$
\left\langle\Psi\left|\hat{N} \hat{\varepsilon}^{v}(x) \hat{\varepsilon}^{v^{\prime}}\left(x^{\prime}\right)\right| \Psi\right\rangle .
$$

In order to calculate this value in forth order of perturbation expansion it should use the Formula (3). But it is not worth to do it. The strait calculation shows that for resonant field scattering $\left(\omega=\omega_{0}\right)$ the construction

$$
\left\langle\Psi_{0}\left|\hat{S}^{(1)+} \hat{N} \hat{A}^{v}(x) \hat{A}^{v}(x) \hat{S}^{(3)}\right| \Psi_{0}\right\rangle,
$$


which appears in such approximation at non-coherent channel results in negative value. This fact evidently contradicts with the positive definition of expression

$$
\left\langle\Psi-f_{0} \psi_{0}\left|\hat{N}\left(\hat{\mathcal{E}}^{v}\right)^{2}\right| \Psi-f_{0} \psi_{0}\right\rangle>0
$$

Such contradiction was found before in Reference [25] where different model has been considered. In order to reconstruct the positive definition of the non-coherent channel using perturbation set it is necessary to average the product $\hat{\mathcal{E}}^{v}(x) \hat{\mathcal{E}}^{v}(x)$ over the wave function $\left(1+\hat{S}^{(1)}+\hat{S}^{(2)}+\hat{S}^{(3)}\right) \Psi_{0}$. But doing this we find the terms proportional to the sixth order of charge. It means that such reconstruction may be achieved only by using higher order terms of perturbation technique. Thus one can not restrict oneself here by the terms of lover order of perturbation technique. So the conventional perturbation theory for $\left\langle\Psi\left|\hat{N} \hat{\mathcal{E}}^{v}(x) \hat{\mathcal{E}}^{v}(x)\right| \Psi\right\rangle$ is problematic. For these reasons we estimate the contribution of non-coherent processes using inequality (2)

$$
\begin{gathered}
\left\langle\Psi-f_{0} \psi_{0}\left|\hat{N} \hat{\mathcal{E}}^{v} \hat{\mathcal{E}}^{v}\right| \Psi-f_{0} \psi_{0}\right\rangle^{2} \geq \\
\left\langle\Psi-f_{0} \psi_{0}\left|\hat{\mathcal{E}}^{v}\right| \Psi-f_{0} \psi_{0}\right\rangle^{2}
\end{gathered}
$$

Then we use the same method to estimate the contribution of coherent channel. Thus according to the quantum theory using Equation (12), Equation (13) and Equation (20) one gets for the scattering by excited atom in two level approximation the following formula;

$$
\left\langle\hat{N} \hat{\mathcal{E}}^{v} \hat{\varepsilon}^{v}\right\rangle_{q u} \geq 2\left|\frac{1}{4 \pi}\left(\frac{e}{m c}\right)^{2} \frac{\left(\delta_{v v_{2}}-n^{v} n^{v_{2}}\right)}{|\mathbf{r}-\mathbf{R}|} a_{\mathbf{k}_{0} \lambda_{0}} e_{\mathbf{k}_{0} \lambda_{0}}^{v_{1}} \sum_{j_{2}} p_{j_{0} j_{2}}^{v_{1}} p_{j_{2} j_{0}}^{v_{2}}\right|^{2} \frac{1}{\left(\hbar \omega_{0}-\hbar c k_{0}\right)^{2}+\frac{\gamma^{2}}{4}}\left(1+\frac{\gamma^{2}}{\left(\hbar \omega_{0}-\hbar c k_{0}\right)^{2}+\frac{\gamma^{2}}{4}}\right)
$$

While according to the semi classical theory one gets

$$
\left\langle\hat{N} \hat{\mathcal{E}}^{v} \hat{\mathcal{E}}^{v}\right\rangle_{s c l} \geq 2\left|\frac{1}{4 \pi}\left(\frac{e}{m c}\right)^{2} \frac{\left(\delta_{v v_{2}}-n^{v} n^{\nu_{2}}\right)}{|\mathbf{r}-\mathbf{R}|} a_{\mathbf{k}_{0} \lambda_{0}} e_{\mathbf{k}_{0} \lambda_{0}}^{v_{1}} \sum_{j_{2}} p_{j_{0} j_{2}}^{v_{1}} p_{j_{2} j_{0}}^{v_{2}}\right|^{2} \frac{1}{\left(\hbar \omega_{0}-\hbar c k_{0}\right)^{2}+\frac{\gamma^{2}}{4}}
$$

The ratio of results of these two calculation methods for the resonant scattering frequency $\omega=\omega_{0}$ is equal to

$$
\frac{\left\langle\hat{N} \hat{\varepsilon}^{v} \hat{\varepsilon}^{v}\right\rangle_{q u}}{\left\langle\hat{N} \hat{\varepsilon}^{v} \hat{\varepsilon}^{v}\right\rangle_{s c l}}=5
$$

The same value characterizes the ratio of scattering cross sections $\sigma_{q u} / \sigma_{s c l}$. This result does not depend on $\gamma$. We note that for the scattering of electromagnetic field on non-excited atoms this ratio is equal to one. The dependence of ratio $\sigma_{q u} / \sigma_{s c l}$ for scattering on excited atom as a function of scattering frequency by $\omega \neq \omega_{0}$ is shown in the Figure 1.

\section{Conclusions}

The evaluations the scattered field amplitude of resonance scattering electromagnetic field on an excited atom can be performed equally well using both the Heisenberg representation and Schroedinger one. In our approximation the both calculations lead to the same results. The same results follow also from the semi-classical theory of radiation, which deals with classical electromagnetic field. In general, the perturbation technique is not suffi- cient to describe the resonance scattering process and we need to sum up the ladder Feynman diagrams. Such procedure is not difficult to be performed using any of theories mentioned above.

In the other case we deal with calculation of the quantum mean values of bilinear products of the field operators $\left\langle\hat{\varepsilon}^{v} \hat{\varepsilon}^{v}\right\rangle$. Here it is more convenient to deal with Sch-

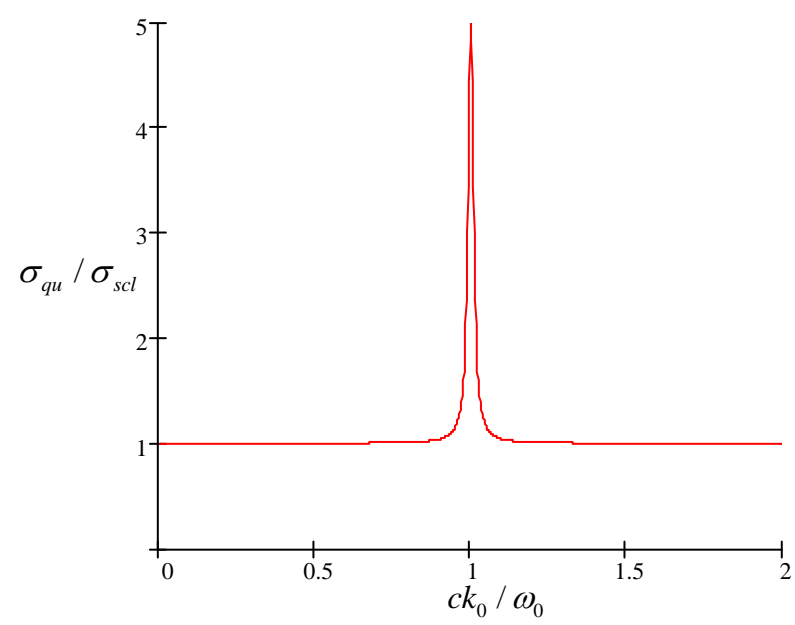

Figure 1. The typical dependence of ratio $\sigma_{q u} / \sigma_{s c l}$ for scattering of electromagnetic field on excited atom as a function of scattering frequency $c k_{0} / \omega_{0}$. 
rödinger representation or with interaction representation, which give additional opportunities to sum up the Feynman diagrams. The letter representations allow us to present the scattering process with the help of two components: coherent (elastic) and non-coherent. Such components could be evaluated independently. The analysis of non-coherent channel shows that the Dyson's summation of ladder Fynman's diagrams by scattering of resonant electromagnetic field on excited atoms is not sufficient. Other summation methods are very unwieldy [8]. In present work we propose the simple method of estimation from below the results of the non-coherent scattering channel. As a result we find that the semi-classical theory of radiation essentially underestimates the cross section of resonance scattering. The quantum theory in its turn shows the violation of equality $\left\langle\hat{N} \hat{\varepsilon}^{v} \hat{\varepsilon}^{v}\right\rangle=$ $\left\langle\hat{\varepsilon}^{v}\right\rangle\left\langle\hat{\varepsilon}^{v}\right\rangle$ in scattered radiation even if such equality took place in the incident electromagnetic field. So the quantum theory results in a change of quantum statistical structure of electromagnetic field due to scattering. This can not be obtained with the help of semi-classical theory of radiation. This change of internal quantum field structure by its scattering on excited atom manifests itself on macroscopic level. Namely such effect makes impossible using here the semi-classical theory of radiation.

\section{References}

[1] W. E. Lamb, “The Theory of Optical Masers," In: C. DeWitt, A. Blandin and C. Cohen-Tannoudji, Eds., Quantum Optics and Electronics, University of Grenoble, Houches, New York-London-Paris, 1965.

[2] M. O. Scully and W. E. Lamb, "Quantum Theory of a Optical Masers,” Physical Review Letters, Vol. 159, No. 2, 1967, pp. 208-226.

[3] C. J. Koester, "9A4-Laser Action by Enhanced Total Internal Reflection,” IEEE Journal of Quantum Electronics, Vol. QE-2, No. 9, 1966, pp. 580-584.
[4] B. B. Boyko and N. S. Petrov, "Reflection of Light from Enhanced and Non Linear Media,” Nauka and Technika, Minsk, 1988.

[5] R. F. Cybulski and C. K. Carniglia, "Internal Reflection from fn Exponential Amplifying Region,” Journal of the Optical Society of America, Vol. 67, No. 12, 1977, pp. 1620-1627.

[6] T. C. Biba, N. S. Petrov and I. Z. Djilavdary, "The Reflection of the Light from Non-Homogeneous Medium with Limited Region of Enhancement," Journal of Applied Spectroscopy, Vol. 32, No. 2, 1980, pp. 226-271.

[7] B. A. Veklenko, "Action of the Noise Processes on Interference Properties in Resonance Spektroscopy,” Izvestija Vouzov Physika, No. 9, 1983, pp. 71-75.

[8] B. A. Veklenko, R. B.Gusarov and Y. B. Sherkunov, "Selective Reflection of Resonance Radiation from Excited Media," Journal of Experimental and Theoretical Physics, Vol. 86, No. 2, 1998, pp. 289-298.

[9] J. Skaar, "Fresnel Function and the Refractive Index of Active Media,” Physical Review E, Vol. 73, No. 2, 2006. pp. 1-7.

[10] I. I. Smolyaninov and Y.-J. Hung, "Enhanced Transmition of Light through a Gold Film due to Excitation of Standing Surface-Plasmon Bloch Waves,” Physical Review B, Vol. 75, No. 3, 2007, pp. 1-4.

[11] T. W. Ebbesen, H. G. Lezec, H. F. Ghaemi, T. Thio and P. A. Wolff. "Extraordinary Optical Transmission through Sub-Wavelength Hole Arrays,” Nature (London), Vol. 391, No. 6668, 1998, pp. 667-669.

[12] V. Weisskopf and E. Wigner, "Berechnung der Naturlichen Linienbreit und Grund der Diracschen Lichttheory," Zeitschrift fur Physik, Vol. 63, No. 1/2, 1930, pp. 54-73.

[13] R. Glauber, “Optical Coherence and Photon Statistics,” In: C. DeWitt. Ed., University of Grenoble, Houches, New York-London-Paris, 1965.

[14] B. A. Veklenko, "Remarks on Kramers-Heisenberg Formula and some Properties of induced Radiation," Izvestija Vuzov. Physika, No. 6, 1987, pp. 132-142. 